\title{
Size-dependent Drug Loading, Gene Complexation, Cell Uptake, and Transfection of a Novel Dendron-Lipid Nanoparticle for Drug/Gene Co-delivery
}

\author{
Ashita Nair ${ }^{1,2 \ddagger}$, Jiyoon Bu ${ }^{1,2 \ddagger}$, Jason Bugno ${ }^{3}$, Piper Rawding ${ }^{1,2}$, Luke Kubiatowicz ${ }^{1,2}$, Woo-jin \\ Jeong ${ }^{1,4}$, and Seungpyo Hong ${ }^{1,2,3,5 *}$ \\ ${ }^{1}$ Division of Pharmaceutical Sciences, School of Pharmacy, University of Wisconsin, Madison, WI 53705, United \\ States \\ ${ }^{2}$ Wisconsin Center for NanoBioSystems (WisCNano), School of Pharmacy, The University of Wisconsin-Madison, \\ 777 Highland Ave., Madison, WI 53705, USA \\ ${ }^{3}$ Department of Biopharmaceutical Sciences, College of Pharmacy, University of Illinois, Chicago, IL 60612, \\ United States \\ ${ }^{4}$ Department of Biological Engineering, Inha University, 100 Inha-ro, Michuhol-gu, Incheon 22212, Republic of \\ Korea \\ ${ }^{5}$ Yonsei Frontier Lab and Department of Pharmacy, Yonsei University, Seoul 03722, Republic of Korea \\ $\$$ These authors equally contributed to this work.
}




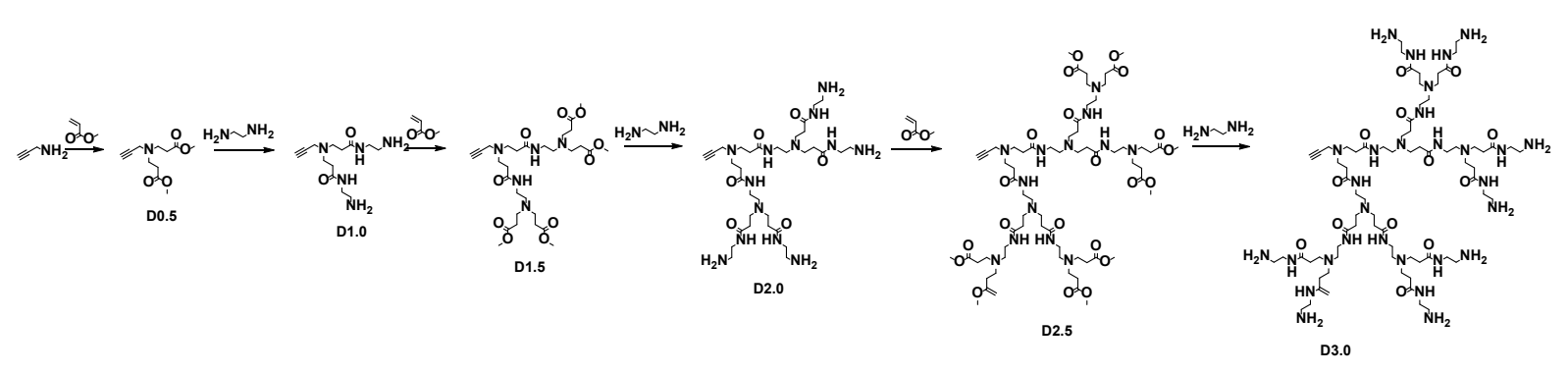

Figure S1. Synthesis of propargyl-focal PAMAM dendrons. 
A)
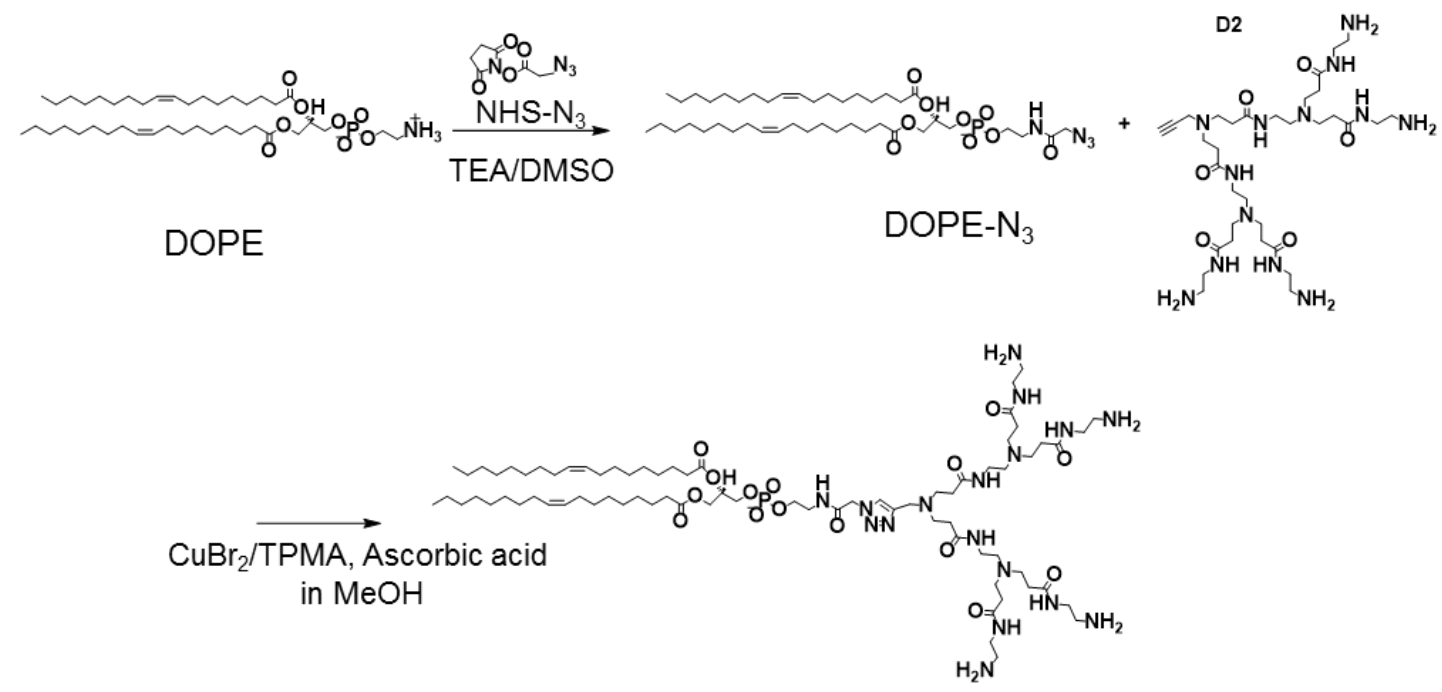

B)

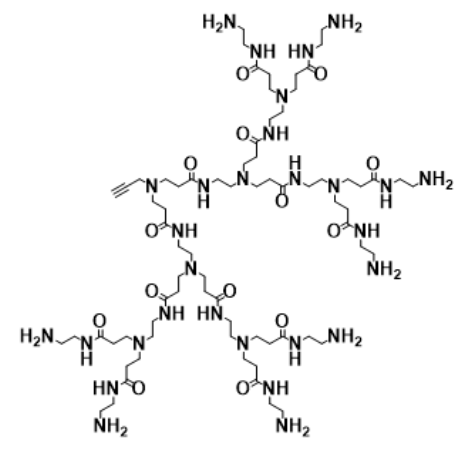

D3

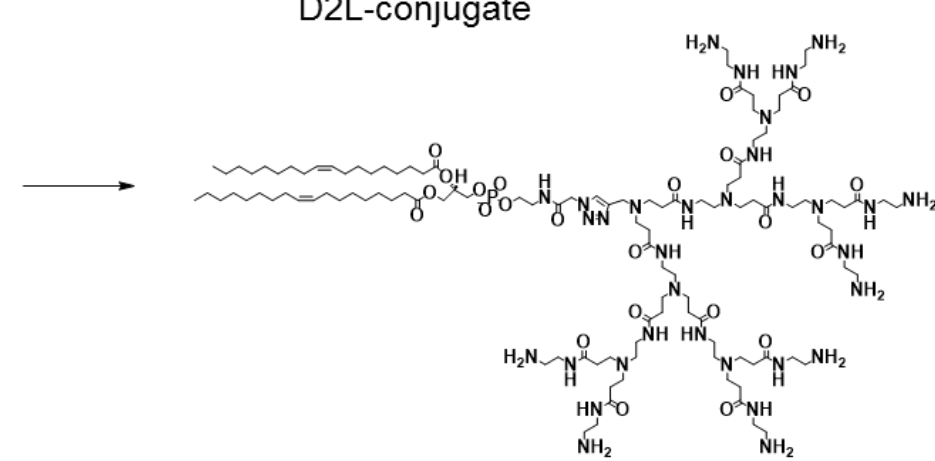

D3L-conjugate

Figure S2. Synthesis of (a) a D2L-conjugate and (b) a D3L-conjugate via 'Click chemistry'. 


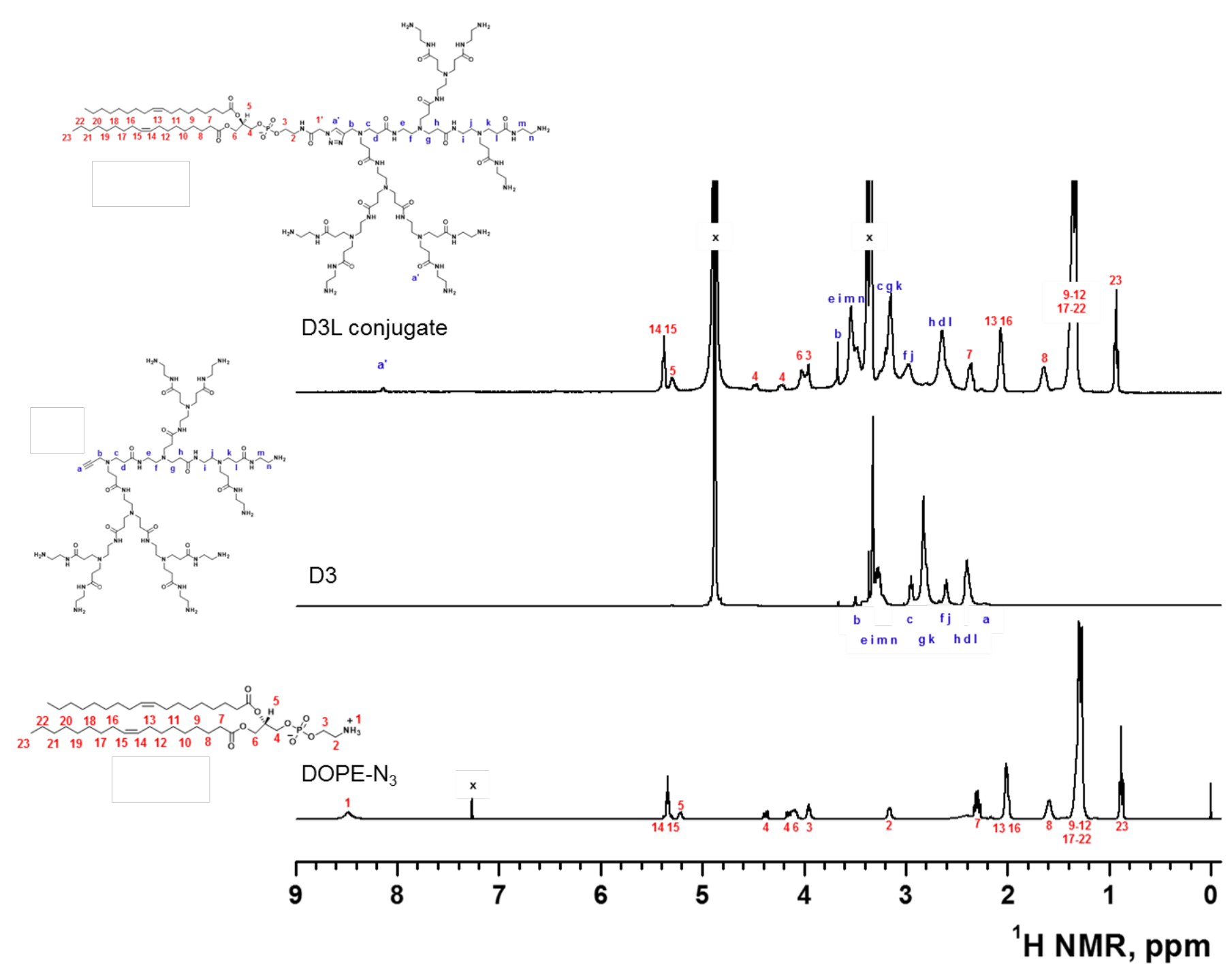

Figure S3. ${ }^{1} \mathrm{H}$ NMR spectrum of DOPE-N3, D3, and D3L-conjugate. 
I: N-H stretch / II: C-H $\left(\mathrm{sp}^{3}\right)$ stretch / IV: $-\mathrm{C}=\mathrm{O}$ (ester) / V: $-\mathrm{C}=\mathrm{O}$ (amide)

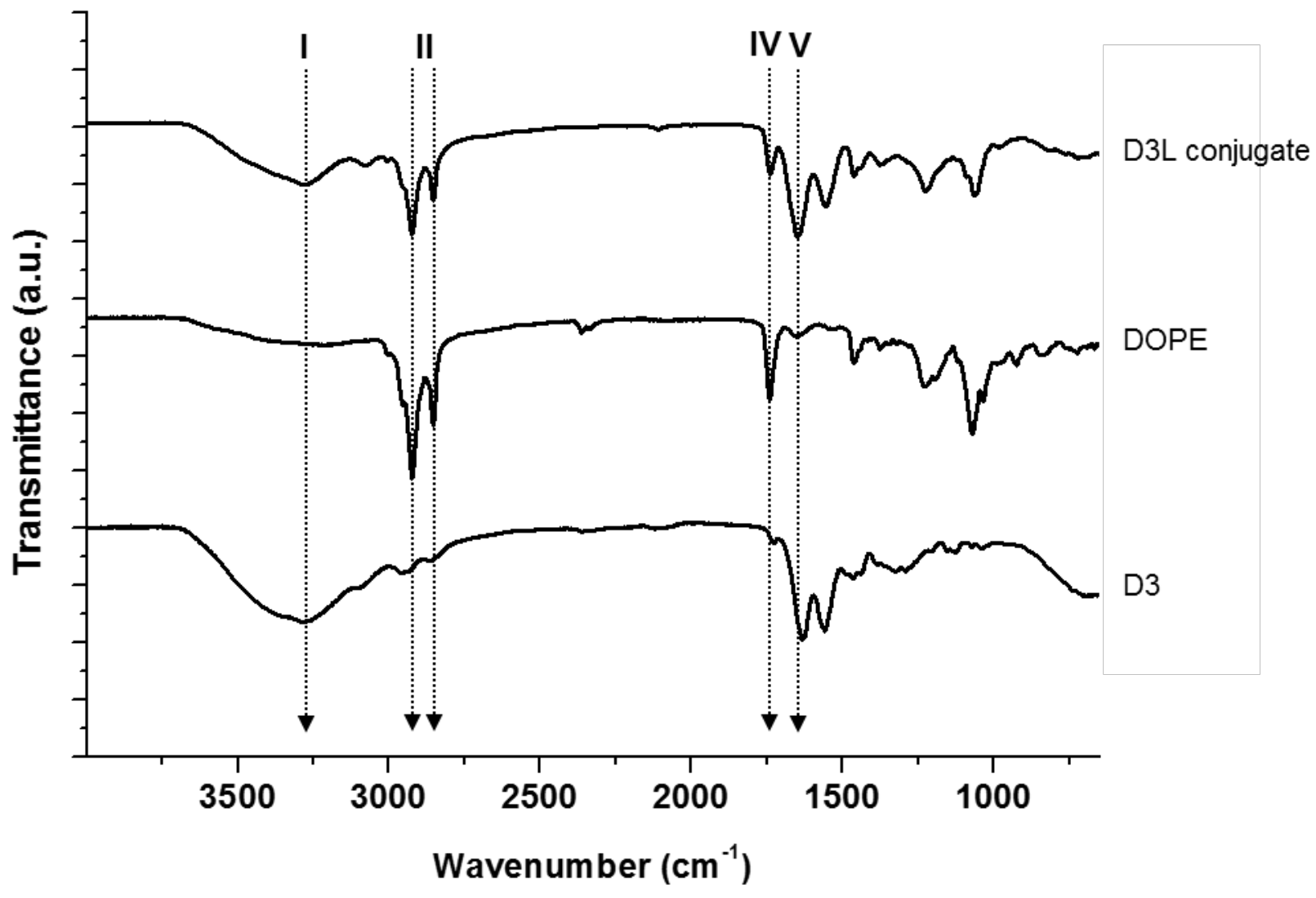

Figure S4. FT-IR spectrum of DOPE, D3, and D3L conjugate. 


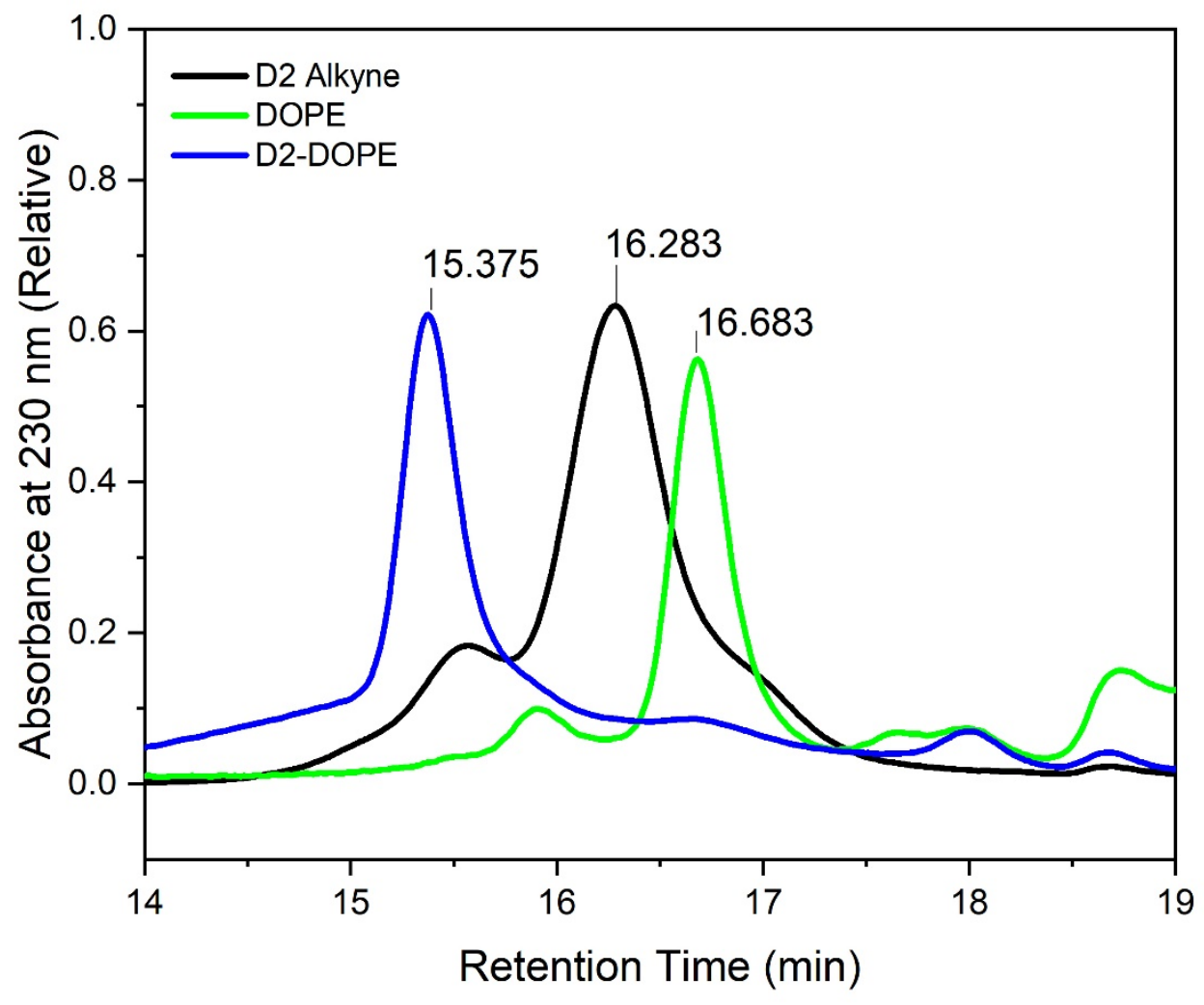

Figure S5. GPC UV absorbance $(\lambda=230 \mathrm{~nm})$ chromatograms of the free dendrons, DOPE, and DLconjugates, confirming the conjugation between the dendron and DOPE. 


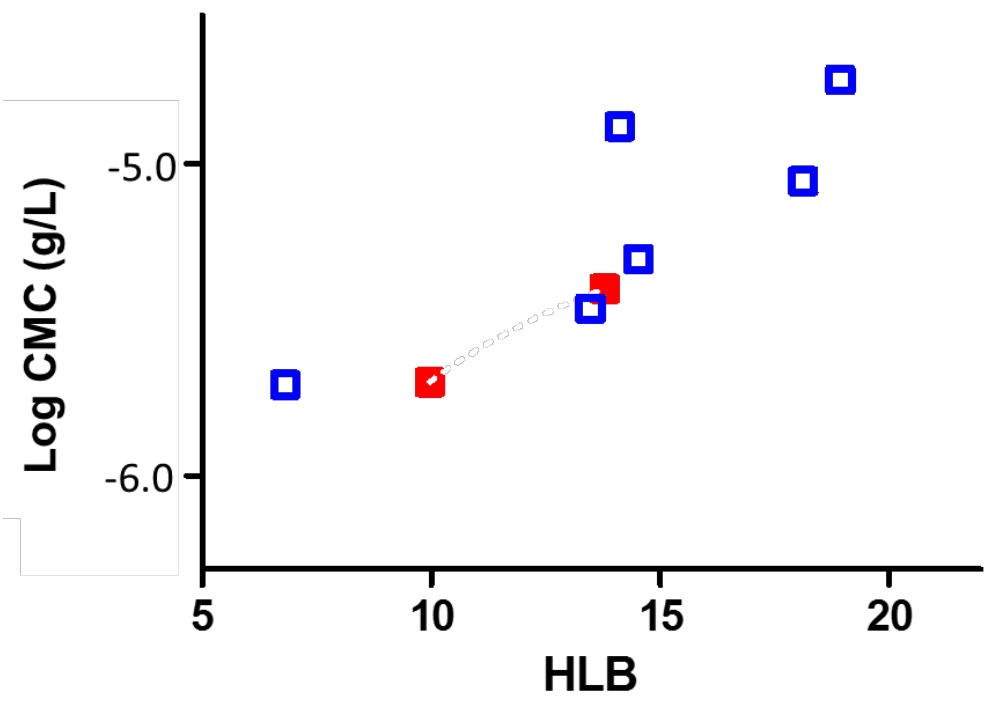

Figure S6. Comparison of CMC values of DLNPs with linear block copolymers having different HLB (DL-NPs: Red, Linear block copolymers: Blue). 


\section{Cholesterol/DL-conjugate ratio}
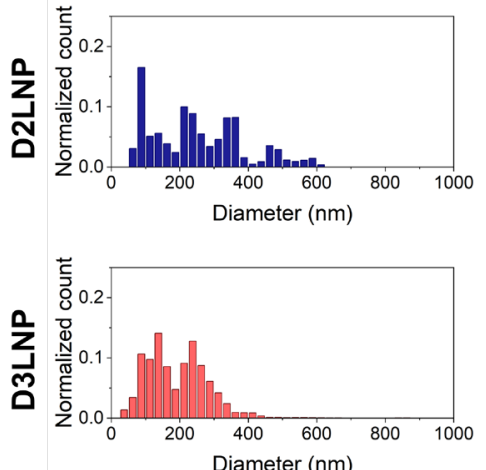
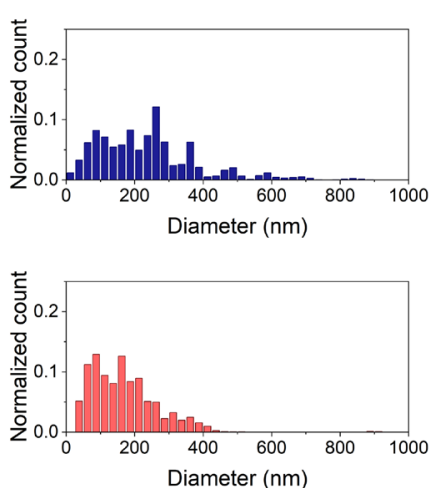

0.5
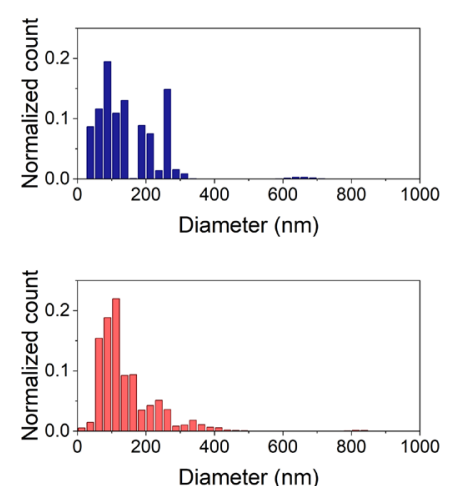
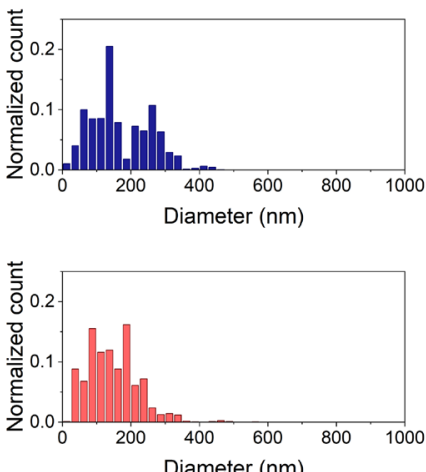
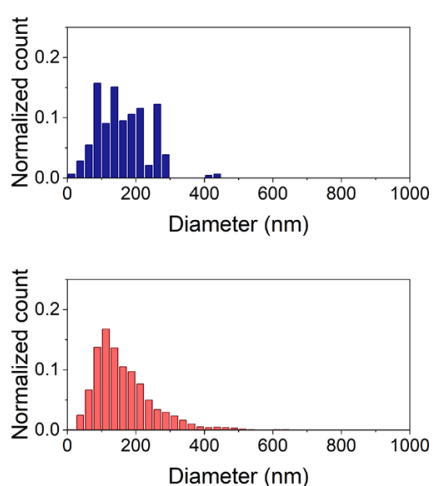

Figure S7. The size distribution of D2LNPs and D3LNPs depending on the molar ratio between cholesterol and DL-conjugates. 


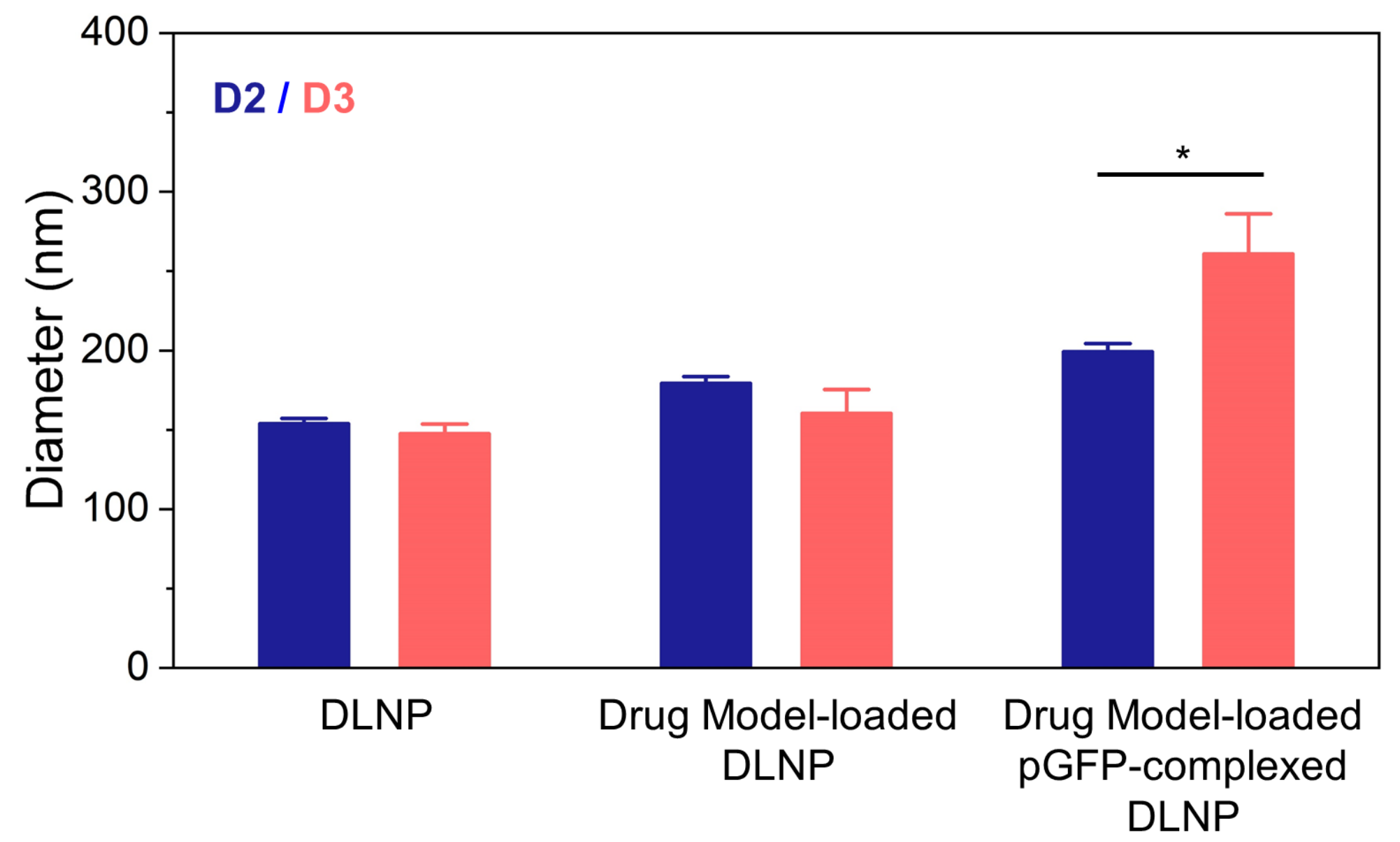

Figure S8. The size of DLNPs after the encapsulation of the drug model and complexation with pGFP. 


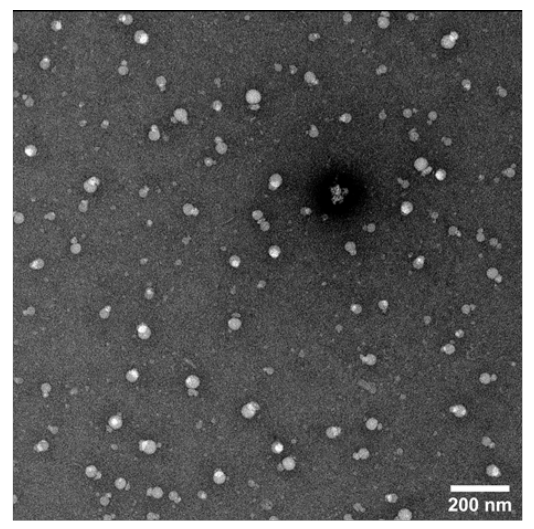

D3LNP

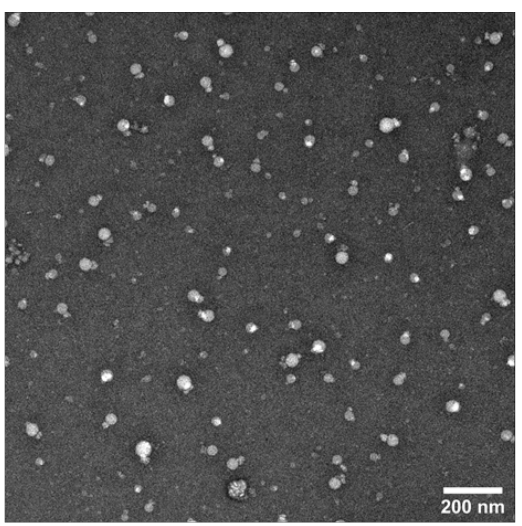

Drug model-loaded D3LNP

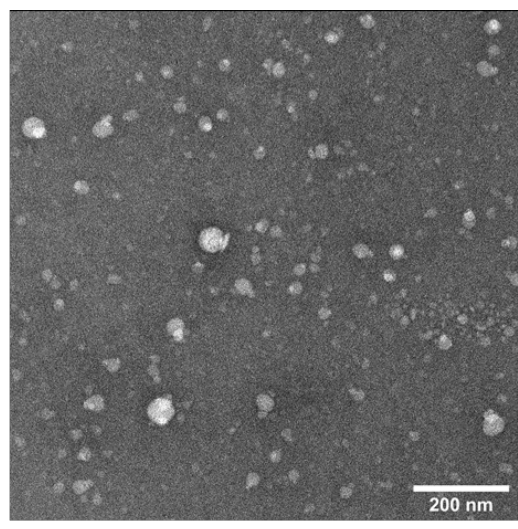

Drug model-loaded pGFP-complexed D3LNP

Figure S9. TEM images (right) of D3LNPs after the encapsulation of the drug model and complexation with pGFP. 

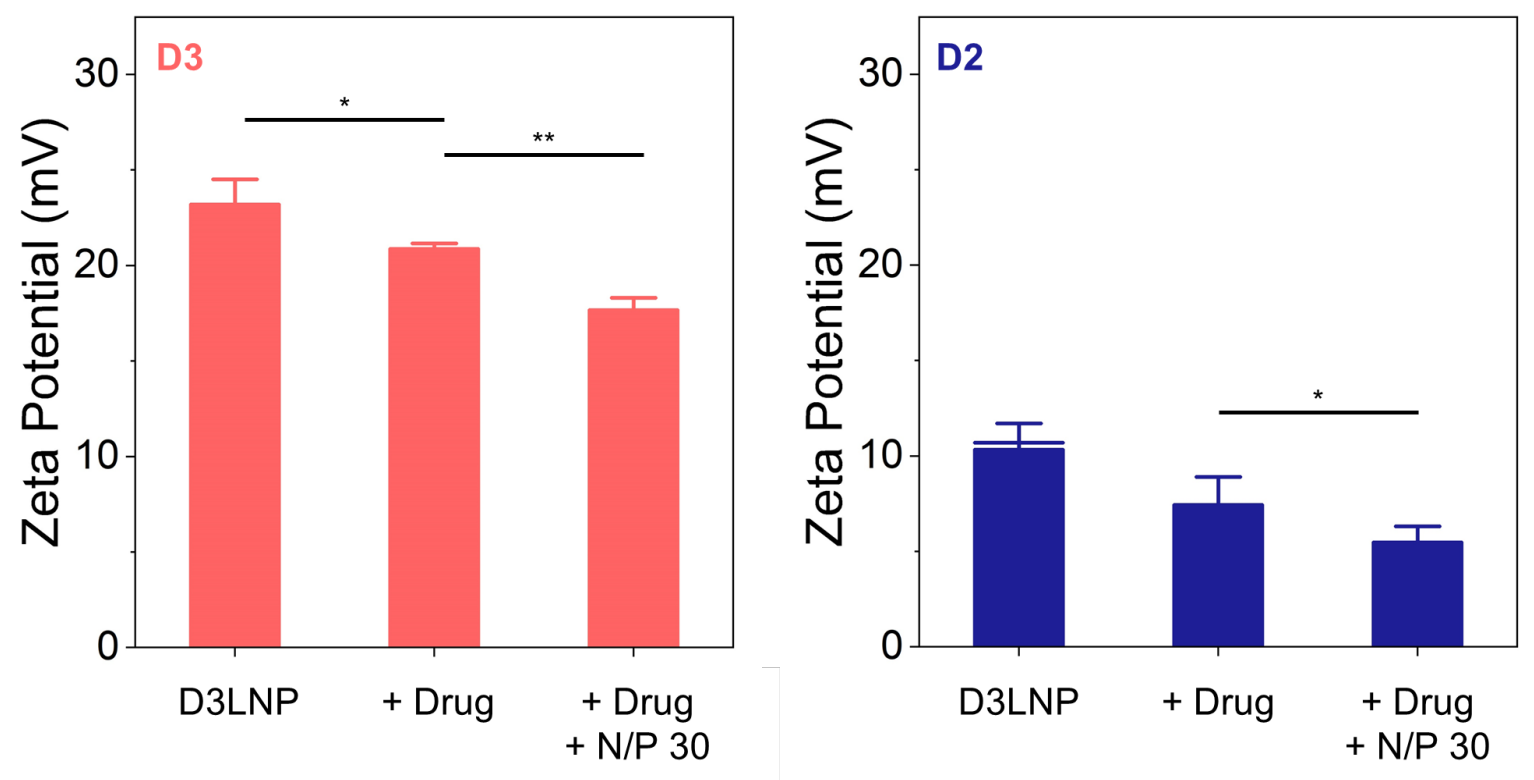

Figure S10. Zeta potentials of DLNPs at cholesterol/DL-conjugate ratio of 0.5. 


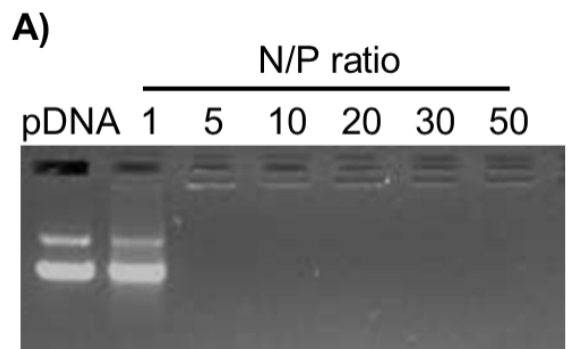

B)

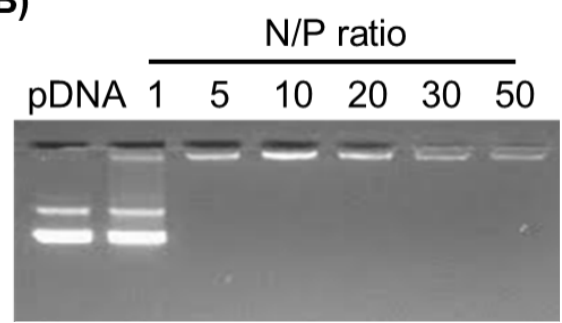

Figure S11. Gel retardation assays of (a) bPEI $25 \mathrm{~K}$ and (b) dendrimer generation 5 at different N/P ratios $(1,5,10,20,30$, and 50). 


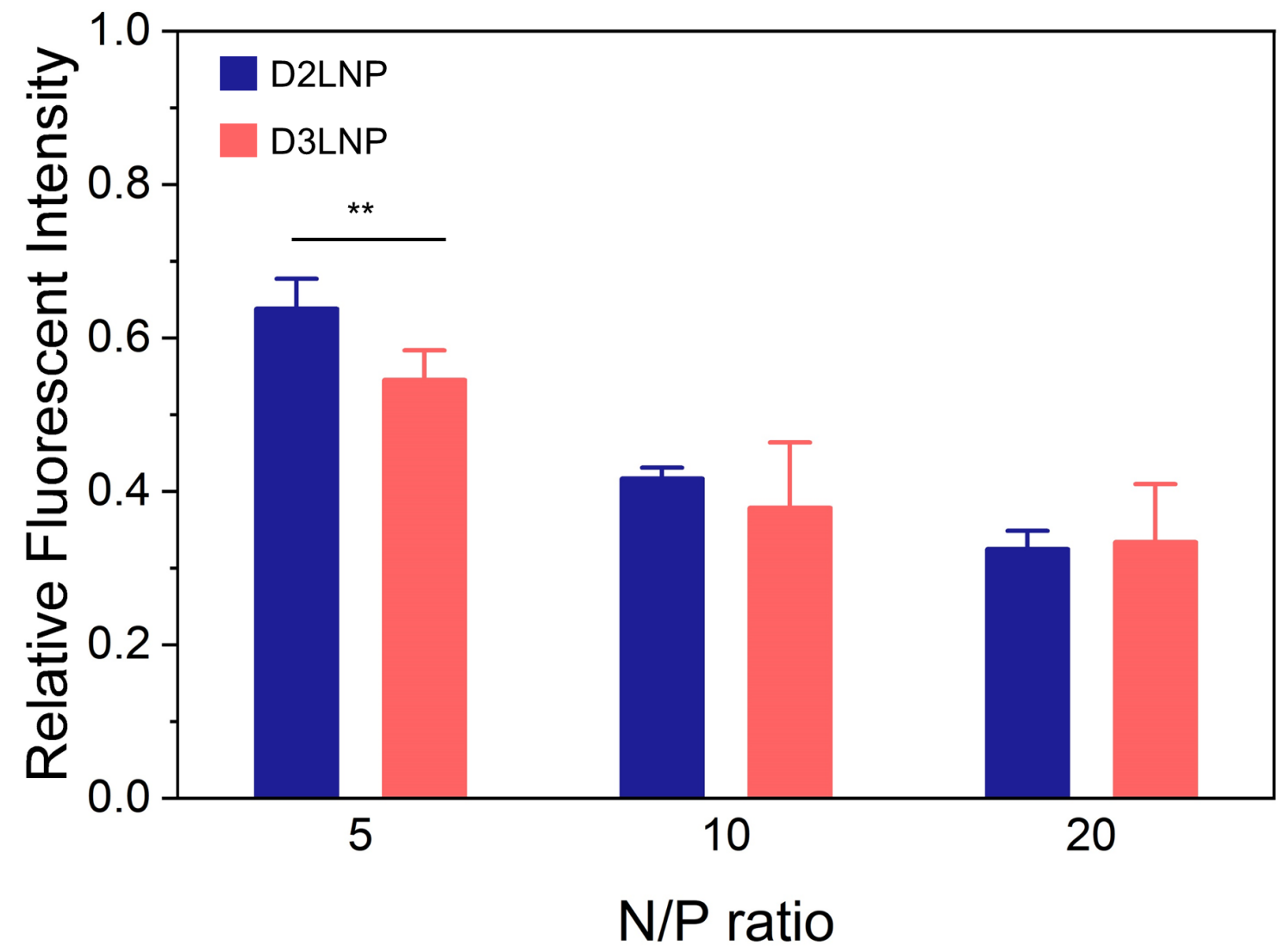

Figure S12. EtBr exclusion assay of D2LNP and D3LNP. 
A)

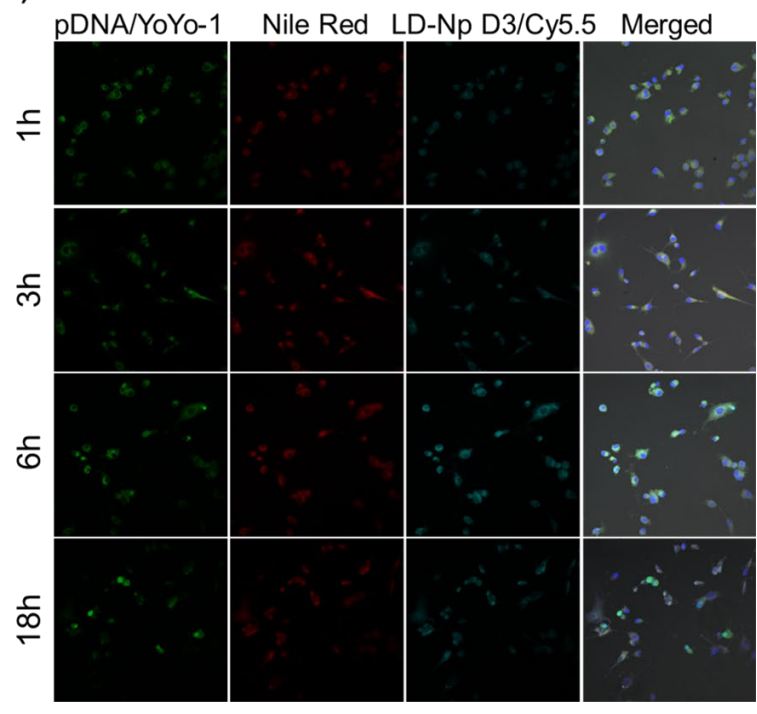

B)

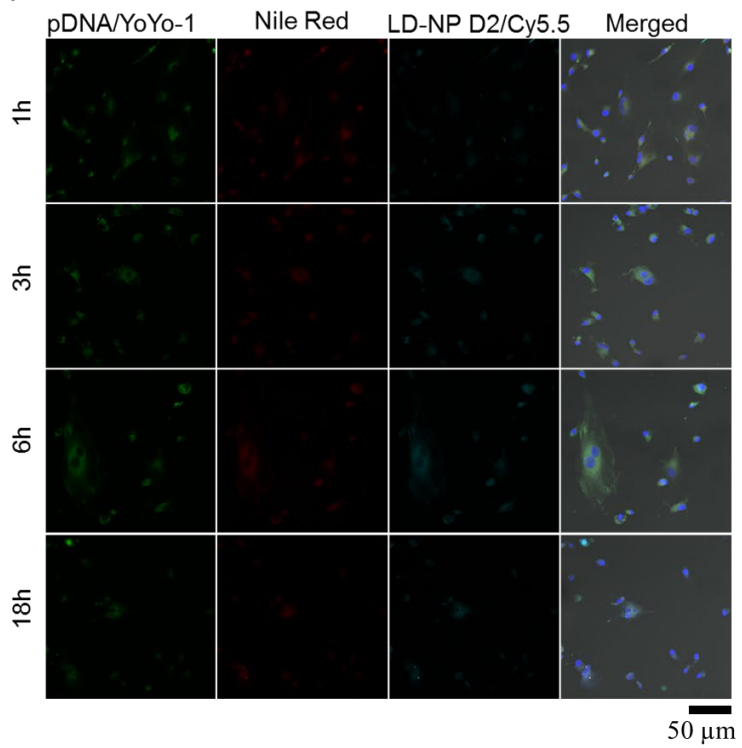

Figure S13. Cellular uptake of pDNA and Nile red loaded (A) D2LNP and (B) D3LNP as a function of time. The pDNA and LDNPs were labeled with YOYO-1 and Cy5.5, respectively. The cellular uptake of each components was monitored by CLSM. 


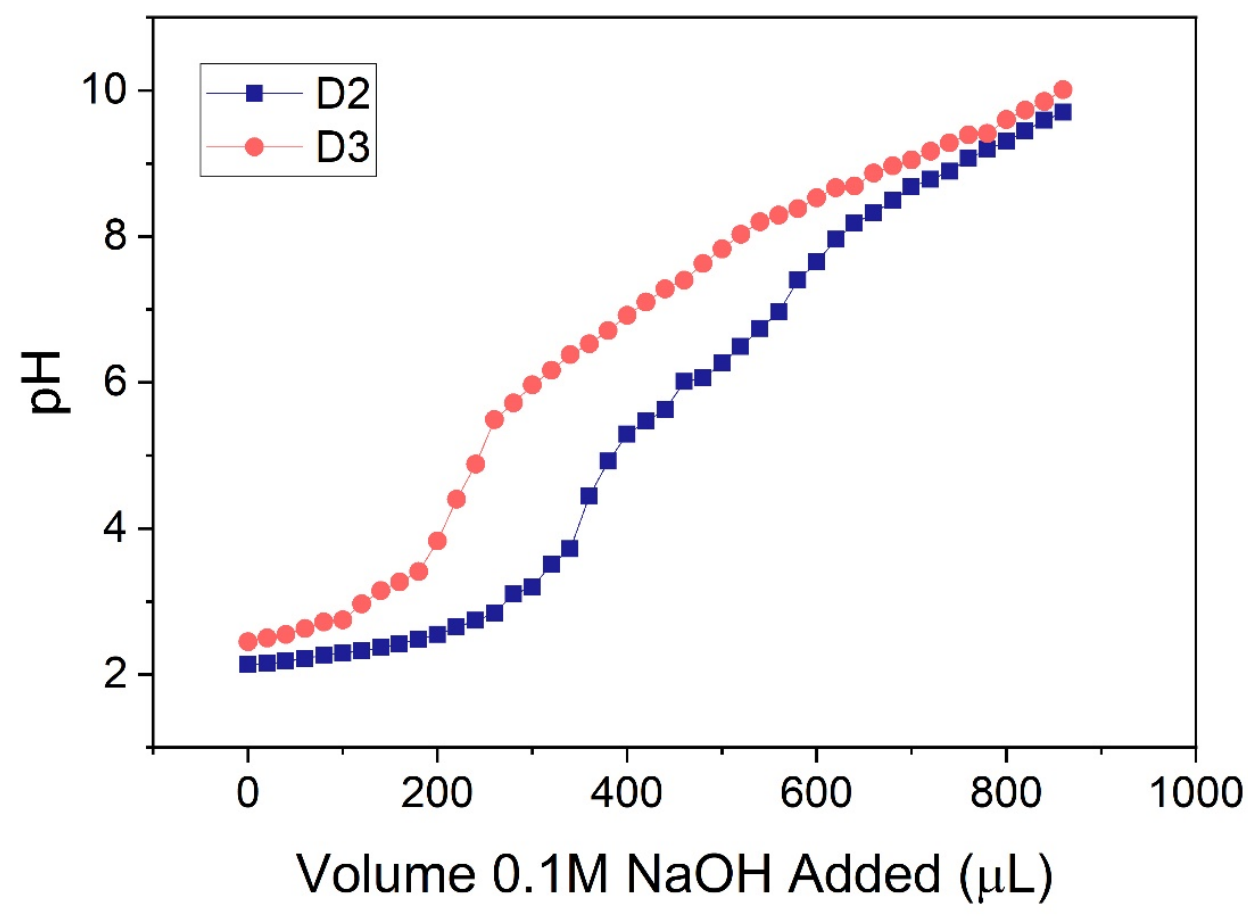

Figure S14. Titration curves of D2 and D3 obtained from potentiometric titrations. 


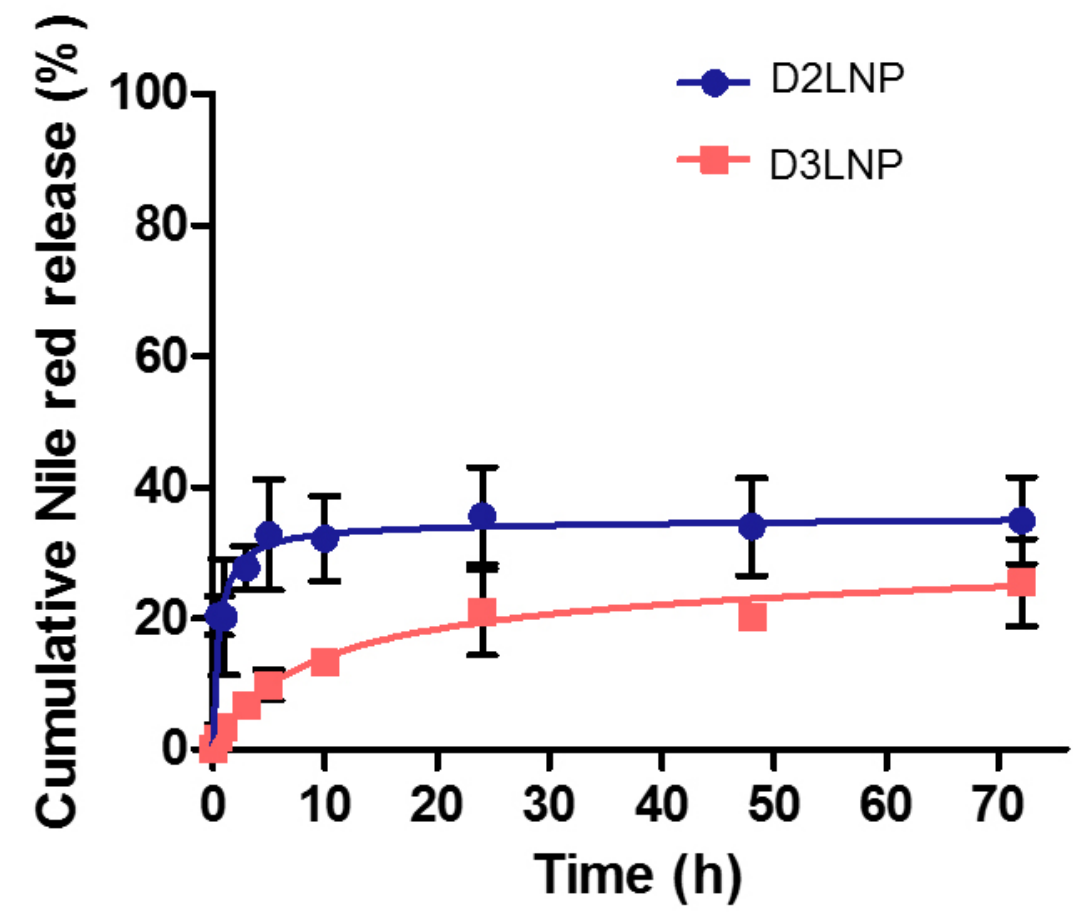

Figure S15. Cumulative Nile red release profiles of D2LNP and D3LNP depending on the incubation time. 


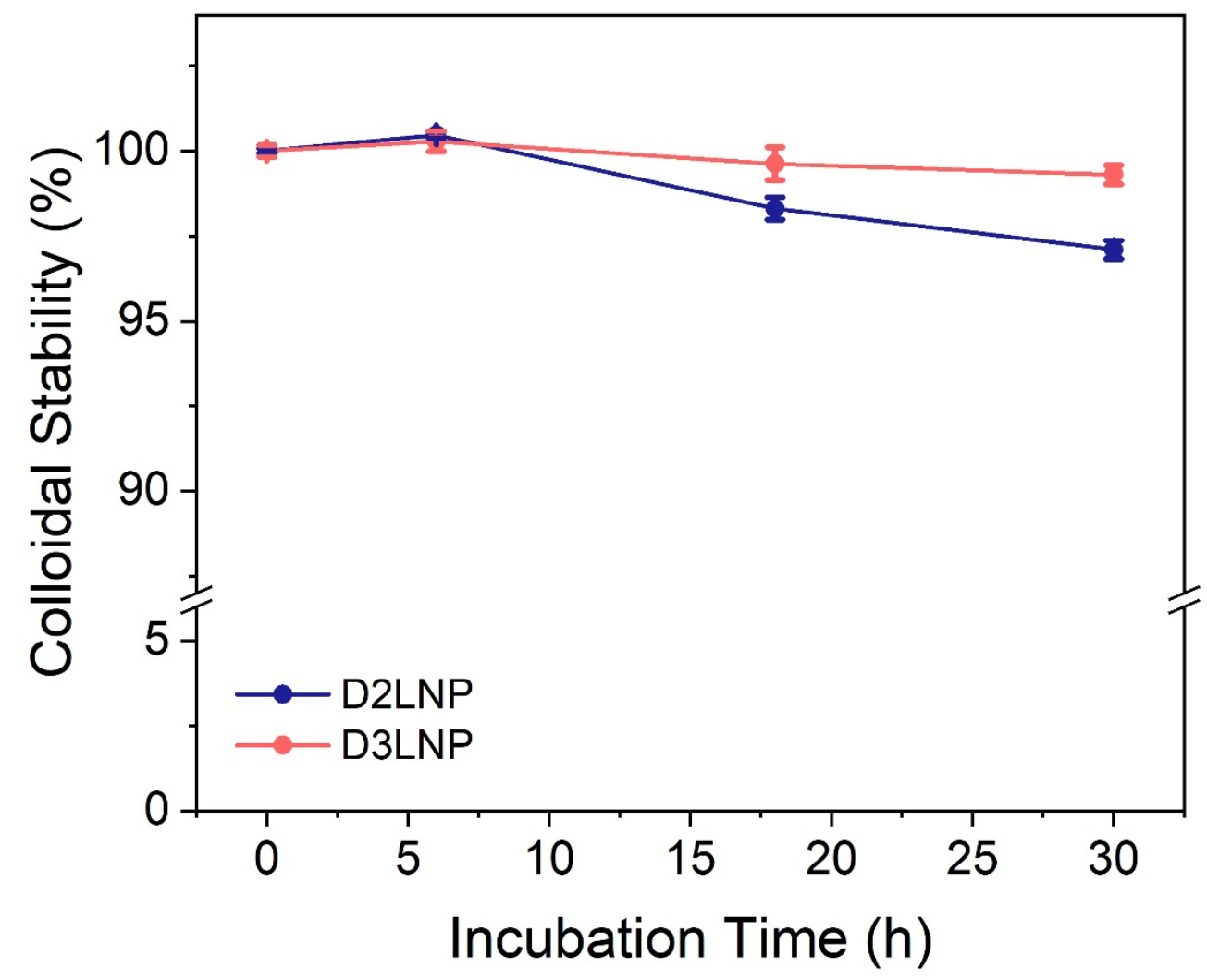

Figure S16. Colloidal stability of DLNPs in PBS solution with 10\% FBS. 


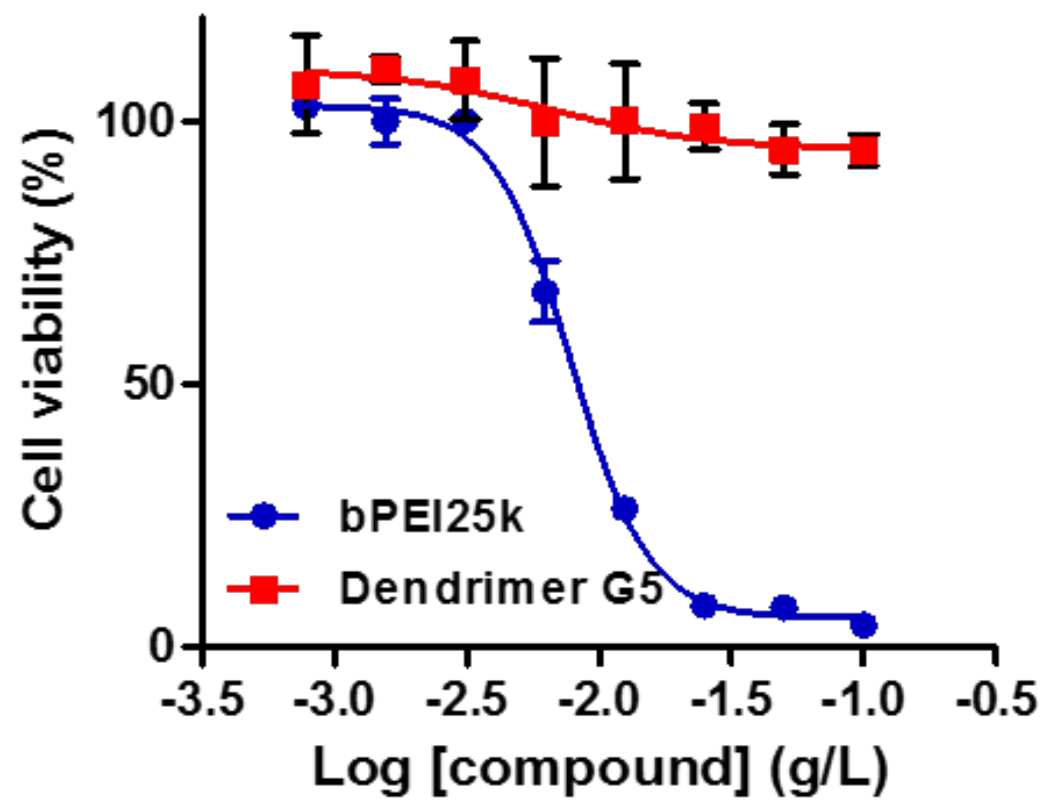

Figure S17. Cell viability determined by MTS assay after treating either bPEI25k or G5 dendrimers. 